\title{
Hemodynamic Profiles of Functional and Dysfunctional Forms of Repetitive Thinking
}

\author{
Cristina Ottaviani, $\mathrm{PhD}^{1}$ (D) Jos F. Brosschot, $\mathrm{PhD}^{2} \cdot$ Antonia Lonigro, $\mathrm{PhD}^{1,3}$. \\ Barbara Medea, $\mathrm{PhD}^{3}$ • Ilse Van Diest, $\mathrm{PhD}^{4}$ • Julian F. Thayer, $\mathrm{PhD}^{5}$
}

(C) The Society of Behavioral Medicine 2016

\begin{abstract}
Background The ability of the human brain to escape the here and now (mind wandering) can take functional (problem solving) and dysfunctional (perseverative cognition) routes. Although it has been proposed that only the latter may act as a mediator of the relationship between stress and cardiovascular disease, both functional and dysfunctional forms of repetitive thinking have been associated with blood pressure (BP) reactivity of the same magnitude. However, a similar $\mathrm{BP}$ reactivity may be caused by different physiological determinants, which may differ in their risk for cardiovascular pathology.

Purpose To examine the way (hemodynamic profile) and the extent (compensation deficit) to which total peripheral resistance and cardiac output compensate for each other in determining BP reactivity during functional and dysfunctional types of repetitive thinking.

Methods Fifty-six healthy participants randomly underwent a perseverative cognition, a mind wandering, and a problem solving induction, each followed by a 5-min recovery period while their cardiovascular parameters were continuously monitored.
\end{abstract}

Cristina Ottaviani

cristina.ottaviani@uniroma1.it

1 Neuroimaging Laboratory, Santa Lucia Foundation, via Ardeatina 306, 00142 Rome, Italy

2 Leiden University, Leiden, The Netherlands

3 Sapienza University of Rome, Rome, Italy

4 University of Leuven, Leuven, Belgium

5 The Ohio State University, Columbus, OH, USA
Results Perseverative cognition and problem solving (but not mind wandering) elicited BP increases of similar magnitude. However, perseverative cognition was characterized by a more vascular (versus myocardial) profile compared to mind wandering and problem solving. As a consequence, $\mathrm{BP}$ recovery was impaired after perseverative cognition compared to the other two conditions.

Conclusions Given that high vascular resistance and delayed recovery are the hallmarks of hypertension the results suggest a potential mechanism through which perseverative cognition may act as a mediator in the relationship between stress and risk for developing precursors to cardiovascular disease.

Keywords Perseverative cognition $\cdot$ Mind wandering . Problem solving $\cdot$ Hemodynamic profile $\cdot$ Cardiac output . Total peripheral resistance

People spend a large part of their time engaging in mind wandering, which often leads to going over the same thoughts again and again, called repetitive thinking. Such an extremely common cognitive process can take functional (problem solving) and dysfunctional (rumination, worry) routes [1]. The perseverative cognition hypothesis specifically suggests that rumination about the past and worrisome thoughts about the future (i.e., perseverative cognition) cause a "fight-or-flight" action tendency, followed by a cascade of biological events such as increases in cardiovascular activity and this persistent physiological activation may have an impact on an individual's health ultimately leading to somatic disease [2, 3]. A recent meta-analysis supported this view, providing evidence of increased cardiovascular, autonomic, and endocrine nervous system activity associated with rumination and worry [4].

However, previous studies repeatedly showed that even ostensibly more functional forms of repetitive thinking, such 
as mind wandering and problem solving could be associated with increased physiological activity. For example, Verkuil and colleagues [5] found cardiac effects of the same magnitude during worrying and problem solving, leading the authors to conclude that "mere mental load may be responsible for at least a part of the physiological effects of worry" (page 448). Similarly, several studies linked mind wandering per se with increased physiological activation [6-8].

If both functional and dysfunctional forms of repetitive thinking were associated with physiological activity, why would only the latter be associated with increased risk for somatic disease? In terms of cardiovascular activity, it has to be noted that elevations in blood pressure (BP) of the same magnitude can be elicited by different patterns of compensatory changes in cardiac output and total peripheral resistance [9]. Therefore, looking at the physiological determinants of $\mathrm{BP}$ becomes more informative than focusing on BP responses per se [10]. The term "hemodynamic profile" describes the relationship between cardiac output and total peripheral resistance in the homeostatic regulation of BP [11]. The first aim of the present study was to examine the hemodynamic profiles of functional and dysfunctional forms of repetitive thinking. We hypothesized that perseverative cognition would be characterized by a predominantly vascular hemodynamic profile whereas problem solving and mind wandering would have a more myocardial or mixed profile. If this were true, then the cardiovascular reactivity of the same magnitude that has been shown to characterize perseverative cognition and problem solving would be associated with distinctive hemodynamic patterns, with different implications for health. In fact, elevated BP driven by total peripheral resistance, compared to cardiac output, has been linked to increased risk of cardiac events and mortality [9, 12, 13].

The second aim of the present study, closely connected to the first, was to show that another crucial difference between functional and dysfunctional forms of repetitive thinking concerns the duration of the concomitant physiological activation. Indeed, only prolonged or chronic activation can lead to the pathogenic state that eventually leads to organic disease [2]. A major consequence of the dominance of reactivity-based theories has been the failure to examine the duration of activation. This is an important limitation considering that a recent metaanalysis showed that poor recovery from laboratory challenges provided incremental value for predicting adverse cardiovascular outcomes beyond reactivity per se [14]. We hypothesized that functional and dysfunctional forms of repetitive thinking would be characterized by an equivalent BP reactivity but only dysfunctional forms would be associated with delayed BP recovery.

The two hypothesis of the present study are closely interconnected. In terms of hemodynamics, delayed recovery has been primarily associated with vascular responding [15]. For example, Steptoe and Marmot [16] found that an increase in
BP over a 3-year period was predicted by impaired post-stress recovery and that the elevation in $\mathrm{BP}$ recorded during the recovery period was determined by vascular rather than cardiac responses. Consistent with this idea, extended mental stress seems to be characterized by transient increases in cardiac output but prolonged changes in total peripheral resistance [17]. Thus, if both our hypothesis are confirmed, it is likely that the delayed recovery that characterizes perseverative cognition would actually be due to its "vascular nature".

In light of the role played by anxiety, depression, and anger-in in augmenting cardiovascular risk [18], the present study also examined which of these personality factors, as well as the dispositional tendency to engage in rumination and worry and state levels of sadness and anxiety, better predicted the hemodynamic profile that characterizes perseverative cognition.

To the best of our knowledge, this is the first study to examine the physiological mechanisms underlying the cardiovascular consequences of functional and dysfunctional forms of repetitive thinking and to examine the hemodynamic mechanisms through how this may ultimately lead to cardiovascular disease.

\section{Method}

\section{Participants}

The sample was composed of university students and employees. Of the 65 subjects who agreed to participate in the study, nine were excluded due to Portapres device (see 'cardiovascular monitoring' below) malfunction. The final sample was composed of 26 women and 30 men with a mean age 24.5 (3.9) years. All subjects were Caucasian. Exclusionary criteria, assessed during a pre-screening questionnaire, were: diagnosis of psychiatric disorders (current and/or past), diagnosis of hypertension or heart disease, any other disease or use of drugs/medications that might affect cardiovascular function, obesity (body mass index $>32 \mathrm{~kg} / \mathrm{m}^{2}$ ), menopause, use of oral contraceptives during the previous 6 months, and pregnancy or childbirth within the last 12 months.

Participants were compensated for their time. The protocol was approved by the Bioethical Committee of S. Lucia Foundation, Rome, Italy.

\section{Procedure}

Participants were informed of the following restrictions: no caffeine, alcohol, nicotine, or strenuous exercise for $2 \mathrm{~h}$ prior to the appointment. After reading and signing the informed consent form, the continuous BP cuff was attached on the middle finger of participants' right hand. After calibration, the experimental protocol started with a 'vanilla' baseline 
period [19]. Then, all participants took part in three experimental conditions: a perseverative cognition induction, a mind wandering induction, and a cognitive problem solving task.

The experimental conditions were presented in counterbalanced order. Each condition lasted $5 \mathrm{~min}$ and was followed by a 5-min recovery period. After baseline, each experimental condition, and each recovery period, participants rated their mood and thoughts by a series of visual analog scales (VAS). Cardiovascular parameters were continuously monitored by the Portapres device throughout the experimental session.

\section{Experimental Manipulation Instructions}

Perseverative cognition induction: "Now I would like you to recall an episode that happened in the past year that made you feel sad, anxious, or stressed, or something that may happen in the future that worries you. Then, I would like you to think about this episode in detail, for example about its possible causes, consequences, and your feelings about it. Please take as much time as you need to identify the event and press the button whenever you are ready".

Mind wandering induction: "Now I would like you to let your mind wander without getting stuck on any particular thought".

Problem solving induction: "Now I would like you to solve a series of syllogisms. Please select "Yes" if you think that the presented syllogism is valid, select "No" otherwise". Example: No A are B. Some C are B. Therefore, some C are not A.

Recovery periods: "The task is terminated. Now I would like you to rest until the instructions for the following task appear on the screen".

\section{Measures}

\section{Cardiovascular Monitoring}

Noninvasive continuous measurement of beat-to-beat BP was obtained throughout the study with the Portapres II (FMS; The Netherlands) device, which has been shown to reliably compare with intra-aortic pressure measurement [20]. The arterial pressure signal was analyzed using BeatScope ${ }^{\mathbb{B}}$ software to obtain systolic (SBP) and diastolic BP (DBP), cardiac output, and total peripheral resistance. SBP, DBP, cardiac output, and total peripheral resistance reactivity values were computed by subtracting the initial baseline from task values. SBP, DBP, cardiac output, and total peripheral resistance recovery values were computed by subtracting task values from posttask values. Hemodynamic profile and compensation deficit were computed as detailed in the following section.

\section{Hemodynamic Profile and Compensation Deficit}

Hemodynamic profile (HP) and compensation deficit were assessed following the orthogonal, physiologically grounded model proposed by Gregg and colleagues [21]. The model is derived from the multiplicative relationship between cardiac output and total peripheral resistance in determining mean arterial pressure [22] and the computation is based on the following equation:

$\log ($ cardiac output $) r+\log ($ total peripheral resistance $) r$

$$
=\log (\text { mean arterial pressure }) r
$$

where $r$ indicates:

a. a ratio of task to baseline values for reactivity periods $[10,21]$;

b. a ratio of recovery to task values for recovery periods [15].

This approach has the advantage of not being based on any artificial taxonomy that would ignore the continuous nature of cardiovascular measurements. The outcome is a continuous variable by which participants are described as more vascular (greater HP values) when the algebraic increase in $\log ($ total peripheral resistance) $r$ exceeds that in $\log$ (cardiac output)r, and more myocardial when the algebraic increase in $\log$ (cardiac output) $r$ exceeds that in $\log$ (total peripheral resistance) $r$. Compensation deficit (CD) increases as the algebraic sum of the $\log$ (cardiac output) $r$ and $\log$ (total peripheral resistance) $r$ values increase [21]. Greater $\mathrm{CD}$ values indicate that increased total peripheral resistance is not compensated by a commensurate decrease in cardiac output.

\section{Visual Analog Scales (VAS)}

After baseline, each task and each recovery period, participants were asked to rate their current levels of feeling anxious, angry, happy, tired, and sad on separate visual analog 100point scales. The same scale was used to inquire about participants' ongoing cognitive activity: "How much for the duration of the task were you": (1) mind wandering, (2) ruminating, (3) worrying. For each VAS, change scores were computed by subtracting the initial baseline from task values for reactivity periods and by subtracting the task from post-task values for recovery periods.

\section{Questionnaires}

Ruminative Response Scale is a measure of depressive rumination tendencies assessed by how often people engage in responses to depressed mood that are self-focused (e.g., I think "Why do I react this way?"), symptom-focused (e.g., I think about how hard it is to concentrate), and focused on the possible consequences and causes of one's mood (e.g., I think "I 
won't be able to do my job if I don't snap out of this") RRS; [23]. Reliability and validity of the RRS were supported through several longitudinal studies with Cronbach's $\alpha$ ranging from 0.75 to $0.80[24,25]$.

Penn State Worry Questionnaire is a 16-item self-report questionnaire mainly focused on future outcomes (e.g., As soon as I finish one task, I start to worry about everything else I have to do) and commonly used to assess pathological worry in both clinical and non-clinical populations PSWQ; [26]. The PSWQ has demonstrated good discriminant validity [27-29] and high internal consistency and test-retest reliability with Cronbach's alphas ranging between .86 and .95 [28].

State Trait Anxiety Inventory includes a measure of trait dispositional anxiety that targets how respondents "generally feel" (e.g., I am a steady person) STAI; [30]. High validity and reliability (Cronbach's alpha from .86 to .95) have been documented [31,32].

Center for Epidemiologic Studies Depression Scale is a 20item self-report scale designed to measure depressive symptomatology (e.g., I felt that everything I did was an effort) over the previous week in the general population CES-D; [33]. The validity of the CES-D has been repeatedly confirmed, although some specific items are currently a matter of debate [34]. Cronbach's alphas are above .85 in the general population and .90 in depressed patients confirming high reliability [33].

Anger-in subscale of the Spielberger Trait Anger Expression Inventory is a measure of the tendency of individuals to hold in or suppress responses to anger provocation (e.g., I control my urge to express my angry feelings) STAXI; [35]. The STAXI has consistently demonstrated evidence to support its validity and reliability as an instrument to assess anger (overall Cronbach's alpha above .90) [36].

\section{Data Analysis}

All data are expressed as means (SD). Differences at $p \leq .05$ were regarded as significant. Data processing was performed with the software modules of SPSS 23 (IBM). SBP, DBP, cardiac output, and total peripheral resistance reactivity and recovery, hemodynamic profile, compensation deficit, and scores on personality questionnaires were treated as continuous variables.

Sex differences were analyzed by $t$ tests and $\chi^{2}$ tests.

To test for differences in reactivity and recovery levels for the three experimental conditions, a series of 3 (induction: perseverative cognition, mind wandering, problem solving) $\times 2$ (time: reactivity, recovery) $\times 6$ (order) general linear models (GLMs) were performed on SBP, DBP, cardiac output, total peripheral resistance, and each VAS. Reactivity and recovery change scores were used in these GLMs.

Consistent with the approach adopted by James and Gregg [37], one-sample $t$ tests were used to test the difference from zero of hemodynamic profile and compensation deficit scores for each condition and subsequent recovery periods. A significant $t$ test result for hemodynamic profile was taken to indicate either a vascular (positive $t$ value) or a myocardial profile (negative $t$ value). A nonsignificant hemodynamic profile result coupled with a significant compensation deficit result means that the response was mixed (i.e., neither vascular nor myocardial). No hemodynamic response at all was deemed to have occurred when both hemodynamic profile and compensation deficit were not significant.

To examine the predictive power of both state moods and dispositional traits in determining the hemodynamic profile that characterizes functional and dysfunctional forms of repetitive thinking above and beyond traditional predictors, three hierarchical multiple regression analyses were conducted with sex entered in the first stage (see below); mood and thought changes (anxious, sad, ruminating, worrying) during the corresponding induction entered in the second stage, and the questionnaires scores entered in the third stage as the independent variables. Hemodynamic profile during the perseverative cognition, mind wandering, and problem solving inductions served as dependent variables.

\section{Results}

\section{Descriptive Statistics}

Significant sex differences emerged for baseline DBP $(t(54)=2.18 ; p=.03)$, and scores on the CES-D $(t(54)=2.91 ; p=.01)$, STAI $(t(54)=3.12 ; p=.003)$, PSWQ $(t(54)=2.65 ; p=.01)$, and $\operatorname{RRS}(t(54)=2.17 ; p=.04)$. As shown in Table 1, men were characterized by higher levels of

Table 1 Sex differences at baseline

\begin{tabular}{llll}
\hline & Women $(n=26)$ & Men $(n=30)$ & $t / \chi^{2}$ \\
\hline Age $($ years $)$ & $24.2(3.4)$ & $24.7(4.5)$ & 0.41 \\
BMI $\left(\mathrm{Kg} / \mathrm{m}^{2}\right)$ & $21.9(2.7)$ & $22.7(4.1)$ & 0.80 \\
Education & $11 \mathrm{~L}, 14 \mathrm{M}, 1 \mathrm{H}$ & $13 \mathrm{~L}, 15 \mathrm{M}, 2 \mathrm{H}$ & 0.25 \\
SBP $(\mathrm{mmHg})$ & $125.7(23.4)$ & $133.7(18.3)$ & 1.44 \\
DBP $(\mathrm{mmHg})$ & $74.5(13.2)$ & $81.8(11.9)$ & $2.18^{*}$ \\
CO $(\mathrm{L} / \mathrm{min})$ & $5.2(1)$ & $5.7(1.2)$ & 1.87 \\
TPR $\left(\mathrm{dyn} / \mathrm{cm}^{2} / \mathrm{s}\right)$ & $1192.3(231)$ & $1152.4(149.7)$ & 0.78 \\
Nicotine & $16 \mathrm{~N}, 10 \mathrm{Y}$ & $24 \mathrm{~N}, 6 \mathrm{Y}$ & 2.33 \\
RRS & $51.5(13.4)$ & $42.7(11.1)$ & $2.17 *$ \\
PSWQ & $43.3(10.3)$ & $54.2(11.9)$ & $2.65^{*}$ \\
STAI & $49.1(10.6)$ & $39.3(8.9)$ & $3.12^{*}$ \\
CES-D & $43.3(10.3)$ & $34.5(8.6)$ & $2.92^{*}$ \\
\hline
\end{tabular}

Note. $B M I$ body mass index, $S B P$ systolic blood pressure, $D B P$ diastolic blood pressure, $C O$ cardiac output, TPR total peripheral resistance, $R R S$ RUMINATIVE RESPONSE SCALE, $P S W Q$ Penn State Worry Questionnaire, STAI STATE TRAIT ANXIETY INVENTORY, CES-D Center for Epidemiologic Studies Depression scale $* p<0.05$ 
DBP and trait worry, whereas women had higher tendencies toward anxiety, depression, and depressive rumination, therefore sex was included as a covariate in all the analyses concerning these variables.

\section{Blood Pressure, Total Peripheral Resistance, and Cardiac Output Reactivity and Recovery}

The GLM having SBP as the dependent variable yielded significant effects of time, $F(1,50)=10.19, p=.002 ; \eta^{2}=.17$, induction $F(2,100)=7.82, p=.001 ; \eta^{2}=.14$, and time $\times$ induction interaction, $F(2,100)=6.20, p=.003 ; \eta^{2}=.11$. As depicted in Fig. 1, post hoc comparisons showed that SBP increases were significantly larger during perseverative cognition and problem solving compared to mind wandering $(p s<.002)$. Moreover, SBP significantly decreased from problem solving to recovery from the same task $(p<.0001)$, but not for mind wandering, and marginally increased from perseverative cognition to the subsequent recovery period $(p=.07)$. Within the recovery period, SBP was higher during perseverative cognition compared to mind wandering and problem solving $(p s<.002)$, with no significant differences between the last two conditions.
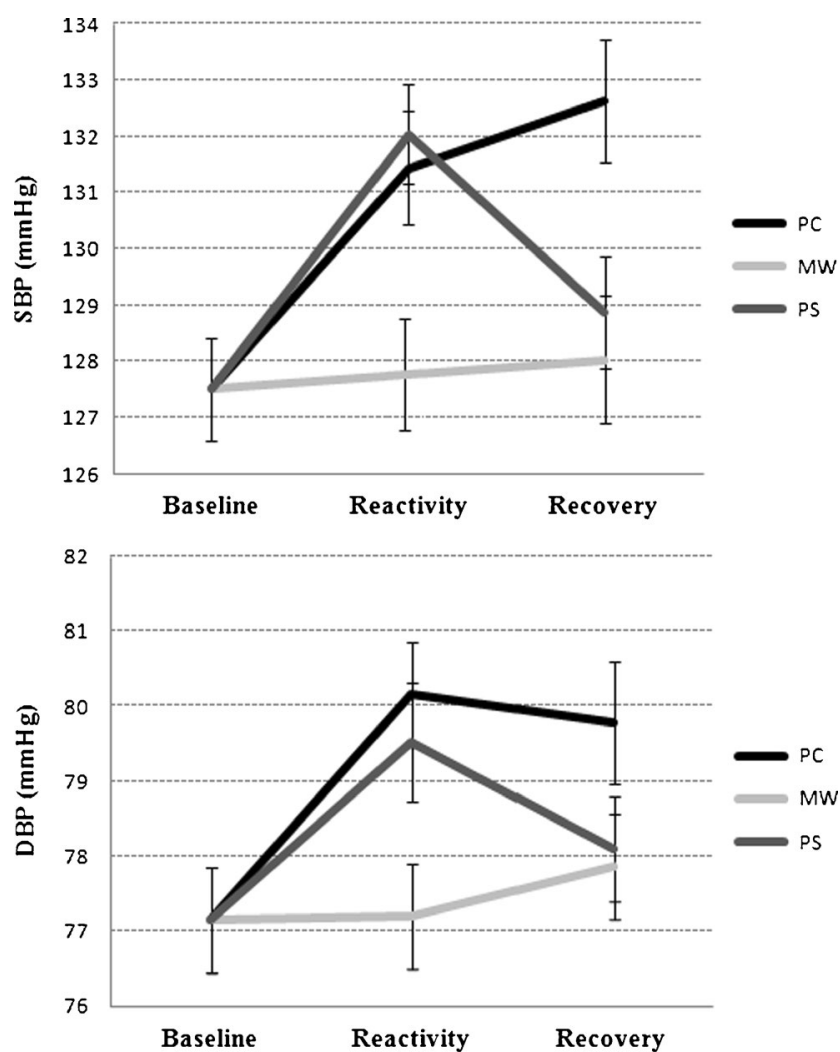

Fig. 1 Systolic and diastolic blood pressure reactivity and recovery for each experimental condition. Note. Error bars represent standard deviations of the mean. $P C$ perseverative cognition, $M W$ mind wandering, $P S$ problem solving, $S B P$ systolic blood pressure, $D B P$ diastolic blood pressure
As for DBP, significant effects of time, $F(1,50)=7.37$, $p=.01 ; \eta^{2}=.13$, induction $F(2,100)=3.83, p=.02$; $\eta^{2}=.07$, and time $\times$ induction interaction, $F(2,100)=5.59$, $p=.005 ; \eta^{2}=.10$ emerged. As illustrated in Fig. 1, during both perseverative cognition and problem solving, there were significantly larger DBP increases compared to mind wandering $(p s<.01)$. Moreover, DBP significantly decreased from problem solving to recovery from the same task $(p=.01)$, but this was not the case for mind wandering and perseverative cognition. Within the recovery period, DBP was higher during perseverative cognition compared to mind wandering and problem solving $(p s<.01)$, with no significant differences between the last two conditions.

The GLM having cardiac output as the dependent variable yielded a marginally significant effect of induction, $F(2,100)=2.68, p=.07 ; \eta^{2}=.05$ with larger increases in cardiac output during problem solving compared to perseverative cognition and mind wandering $(p s<.02)$ and during mind wandering compared to perseverative cognition $(p=.08)$.

As for total peripheral resistance, significant effects of time, $F(1,50)=5.97, p=.02 ; \eta^{2}=.11$, induction $F(2,100)=26.53$, $p<.0001 ; \eta^{2}=.35$, and time $\times$ induction interaction, $F(2,100)=3.93, p=.02 ; \eta^{2}=.07$ emerged. Post hoc comparisons showed that total peripheral resistance increased more during perseverative cognition compared to mind wandering and problem solving $(p s<.0001)$ with no differences between the last two conditions. A marginally significant result emerged for the recovery phase, with higher total peripheral resistance after perseverative cognition compared to the other two inductions $(p=.068)$.

\section{Hemodynamic Profile and Compensation Deficit}

Figure 2 shows the relationship between hemodynamic profile and compensation deficit scores for the different tasks. No significant sex differences emerged in the hemodynamic profile induced by the different experimental conditions. Onesample $t$ tests for hemodynamic profile and compensation deficit indicated that a vascular profile was produced by the perseverative cognition induction, $t(55)=3.85, p<.0001$, whereas the problem solving condition evoked a myocardial profile, $t(55)=-3.35, p<.0001$, and no hemodynamic response occurred during the mind wandering induction, $t(55)=0.02, p=.98$. A significant compensation deficit emerged during both the perseverative cognition $(t(55)=6.64, p<.0001)$, and the problem solving $(t(55)=5.86, p<.0001)$ - but not during the mind wandering-inductions providing a potential explanation for the increase in BP that characterized these two tasks.

When recovery after the perseverative cognition induction was examined, no hemodynamic response seemed to occur in the transition from reactivity to recovery $(t(55)=-0.42$, 


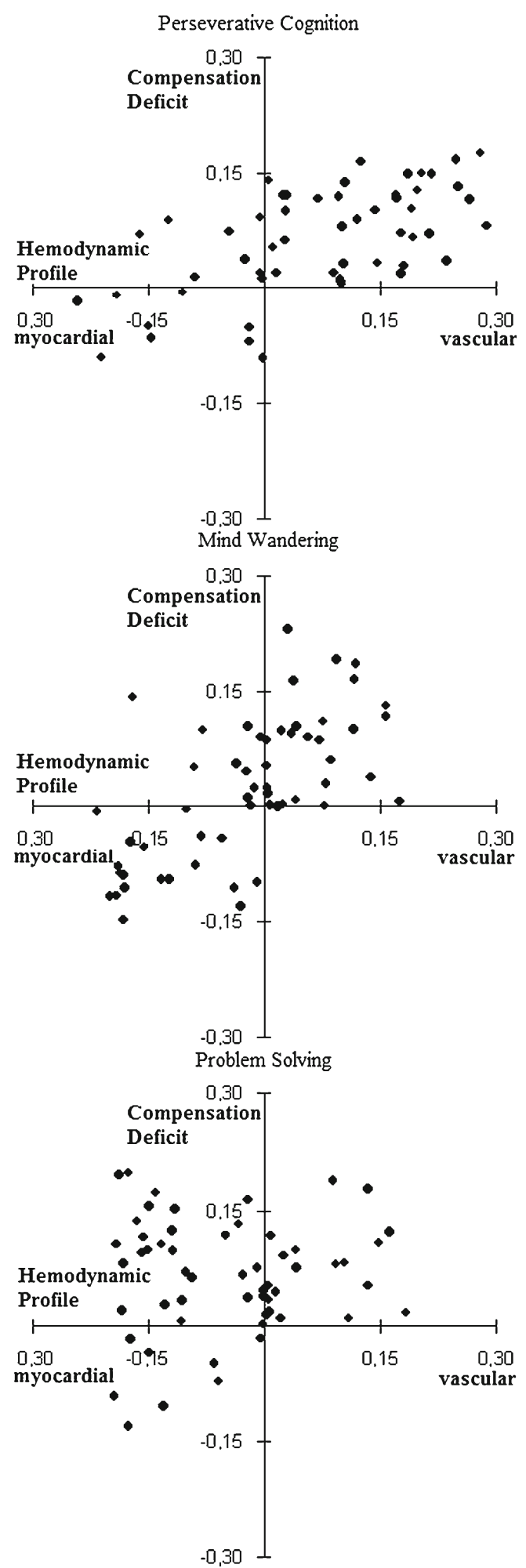

Fig. 2 Scatterplots for hemodynamic profile and compensation deficit during each task. A "more vascular" profile is associated with more positive values along the hemodynamic profile axis and a "more myocardial" profile is associated with more negative values along the hemodynamic profile axis. A "higher deficit" in compensating is associated with more positive values on the compensation deficit axis and a "lower deficit" in compensating is associated with more negative values on the compensation deficit axis $p=.68$ and $t(55)=0.57, p=.57$ for hemodynamic profile and compensation deficit, respectively), indicating that the vascular profile provoked by the induction did not change during the subsequent recovery period. A mixed profile characterized the transition from reactivity to recovery periods for both mind wandering $(t(55)=0.06, p=.96$ and $t(55)=2.90, p=.005$ for hemodynamic profile and compensation deficit, respectively) and problem solving $(t(55)=1.03, p=.31$ and $t(55)=3.14$, $p=.003$ for hemodynamic profile and compensation deficit, respectively).

\section{VAS}

To control for violations of sphericity degrees of freedom were corrected using the Greenhouse-Geisser estimates of epsilon.

As shown in Fig. 3 and Table 2, self-reported levels of mind wandering, rumination, and worry confirmed effectiveness of the experimental manipulations. For the GLM having mind wandering as the dependent variable, main effects of time, $F(1,50)=29.08, p<.0001, \eta^{2}=.37$, and induction $F(2,100)=7.57, p=.005$, epsilon $=.60, \eta^{2}=.13$, were qualified by an interaction between time and induction, $F(2,100)=21.71, p<.0001$, epsilon $=.82, \eta^{2}=.30$. A significant main effect of task order, $F(5,50)=3.19, p=.01$, $\eta^{2}=.24$ and a task order $\times$ induction interaction, $F(10,100)=3.25, p=.001, \eta^{2}=.25$ also emerged. As to rumination, significant main effects of time, $F(1,50)=14.65, p<.0001, \eta^{2}=.23$, and induction, $F(2,100)=89, p<.0001$, epsilon $=.54, \eta^{2}=.64$, and time $\times$ induction interaction $(F(2,100)=84.51, p<.0001$, epsilon $=.74, \eta^{2}=.63$ ) emerged. For worry, the GLM yielded a main effect of induction, $F(2,100)=26.31, p<.0001$, epsilon $=.55, \eta^{2}=.35$ and a significant time $\times$ induction interaction, $F(2,100)=46.83, p<.0001$, epsilon $=.77, \eta^{2}=.48$. Figure 3 illustrates significant post hoc comparisons.

As to self-reported mood (see Table 2 for means and standard deviations and Fig. 3 for significant post hoc results), the GLM having angry as a dependent variable revealed main effects of time, $F(1,50)=25.32, p<.0001, \eta^{2}=.34$, and induction $F(2,100)=42.52, p<.0001$, epsilon $=.54$, $\eta^{2}=.46$, qualified by an interaction between time and induction, $F(2,100)=25.52, p<.0001$, epsilon $=.76, \eta^{2}=.34$.

For anxious, a significant effect of induction, $F(2,100)=5.46, p=.01$, epsilon $=.55, \eta^{2}=.10$, and a time $\times$ induction interaction, $F(2,100)=14.11, p<.0001$, epsilon $=.81, \eta^{2}=.22$ emerged, with higher increases during perseverative cognition compared to the other two inductions.

No significant effects emerged for tired.

The GLM having happy as a dependent variable revealed main effects of time, $F(1,50)=12.28, p=.001, \eta^{2}=.20$, and induction $F(2,100)=14.60, p<.0001$, epsilon $=.55, \eta^{2}=.23$, qualified by an interaction between time and induction, $F(2,100)=4.80, p=.01$, epsilon $=.79, \eta^{2}=.09$. 


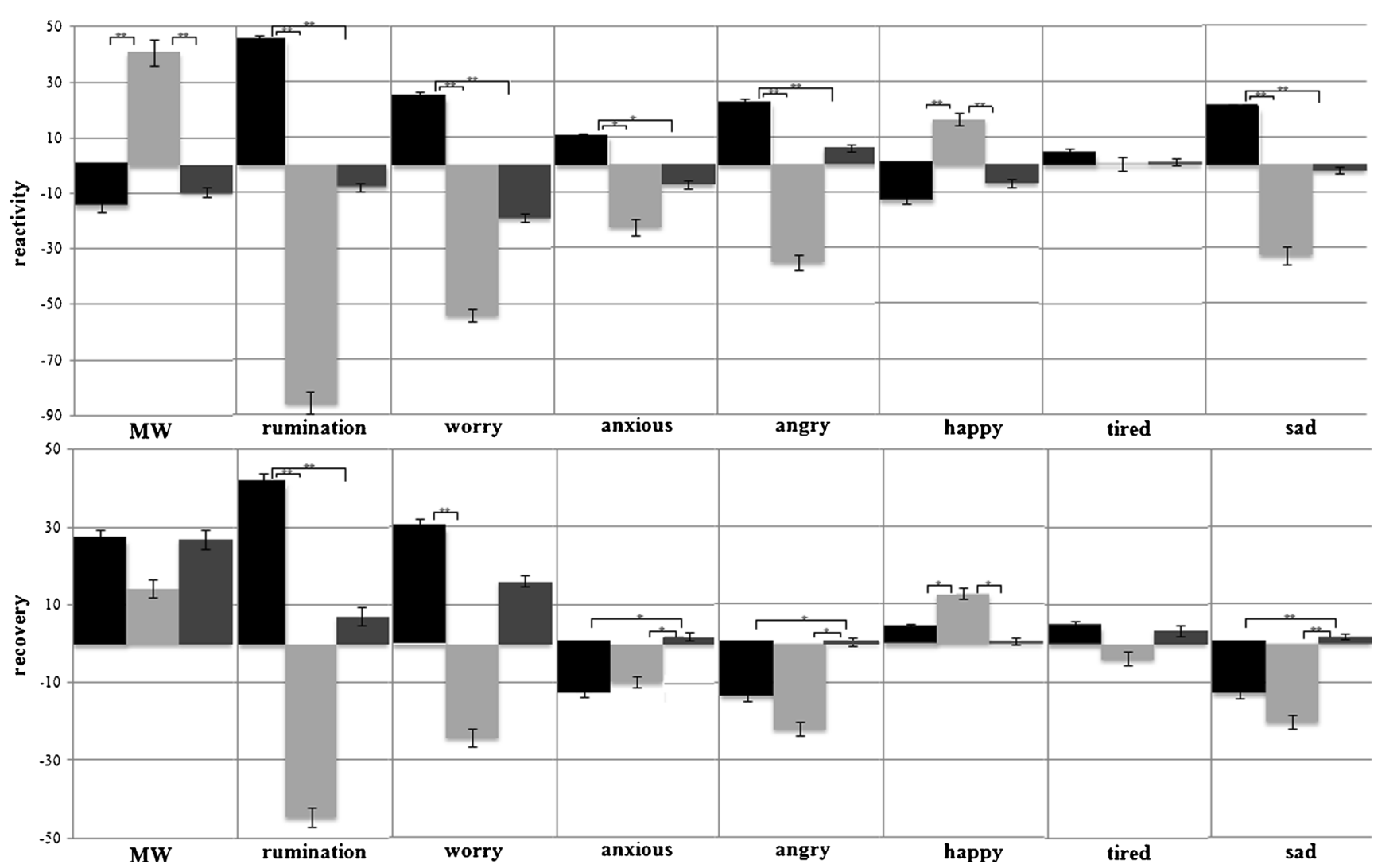

Fig. 3 VAS reactivity and recovery change scores for perseverative cognition (black), mind wandering (light gray), and problem solving

(dark gray). Note. Error bars represent standard deviations of the mean. ${ }^{*} p<.05 ; * * p<.0001 . M W$ mind wandering

As to sad, the GLM revealed main effects of time, $F(1,50)=6.98, p=.01, \eta^{2}=.12$, induction $F(2,100)=26.25$, $p<.0001$, epsilon $=.60, \eta^{2}=.34$, and time $\times$ induction interaction, $F(2,100)=21.92, p<.0001$, epsilon $=.73, \eta^{2}=.31$.

Overall, participants reported to be angrier, sadder, and more anxious during the perseverative cognition induction compared to the mind wandering and problem solving inductions.

\section{Hierarchical Regression}

Given the high correlation between RRS and CES-D scores $(r=.81 ; p<.0001)$ and between PSWQ and STAI $(r=.77$; $p<.0001$ ) scores, only STAI, CES-D, and STAXI-in were included as predictors, to prevent multicollinearity. For the same reason, only sad and anxious (correlations with rumination: $r=.31$; $p=.02$ and $r=.50 ; p<.0001$, respectively) were included in the models.

Results from the hierarchical regression for the prediction of hemodynamic profile during perseverative cognition did not reveal a significant relationship between sex and the dependent variable $\left(\beta=.08 ; p=.65 ; R^{2}=.006\right)$. Momentary mood changes significantly added to the prediction with a higher anxiety response to the perseverative cognition induction being a significant predictor of a more vascular profile during perseverative
Table 2 Means and standard deviations of VAS scores during the three experimental conditions (reactivity) and subsequent recovery periods

\begin{tabular}{|c|c|c|c|c|c|c|}
\hline & \multicolumn{2}{|c|}{ Perseverative cognition } & \multicolumn{2}{|c|}{ Mind wandering } & \multicolumn{2}{|c|}{ Problem solving } \\
\hline & Reactivity & Recovery & Reactivity & Recovery & Reactivity & Recovery \\
\hline Ruminating & $44.6(33.4)$ & $41.2(35.0)$ & $-85.8(60.4)$ & $-44.6(33.4)$ & $-7.9(24.2)$ & $6.9(27.2)$ \\
\hline Worrying & $24.2(35.1)$ & $29.9(34.5)$ & $-54.1(62.9)$ & $-24.2(35.1)$ & $-19.1(28.4)$ & $16.0(29.7)$ \\
\hline Wandering & $-14.2(36.7)$ & $26.6(39.3)$ & $40.8(70.1)$ & $14.2(36.7)$ & $-9.5(26.9)$ & $26.8(35.8)$ \\
\hline Happy & $-12.6(21.4)$ & $3.8(18.9)$ & $16.4(34.29)$ & $12.6(21.4)$ & $-6.7(21.9)$ & $0.5(15.7)$ \\
\hline Sad & $20.2(27.2)$ & $-12.6(26.3)$ & $-32.8(47.1)$ & $-20.2(27.2)$ & $-2.1(19.7)$ & $1.8(11.9)$ \\
\hline Tired & $3.9(29.3)$ & $4.5(17.4)$ & $0.6(38.8)$ & $-3.9(29.3)$ & $1.1(19.6)$ & $3.4(14.9)$ \\
\hline Anxious & $9.9(28.2)$ & $-12.5(23.3)$ & $-22.4(45.0)$ & $-9.9(28.2)$ & $-7.2(23.9)$ & $1.7(17.1)$ \\
\hline Angry & $22.0(25.4)$ & $-13.3(23.5)$ & $-35.3(43.6)$ & $-22.0(25.4)$ & $6.1(20.3)$ & $0.4(16.5)$ \\
\hline
\end{tabular}


cognition $\left(\beta=.41 ; p=.04 ; R^{2}=.10\right)$. Dispositional traits further added to the prediction of hemodynamic profile during perseverative cognition, particularly with higher anger-in scores being associated with a more vascular profile during perseverative cognition, $\beta=.35 ; p=.04 ; R^{2}=.28$.

Neither sex $\left(\beta=-.05 ; p=.77 ; R^{2}=.01\right)$, nor momentary mood changes (sad, $\beta=-.13 ; p=.47$; anxious, $\beta=.08$; $p=.67 ; R^{2}=.02$ ) were significant predictors of hemodynamic profile during problem solving. Among dispositional traits, higher anger-in scores significantly predicted a more vascular profile during problem solving, $\beta=.38 ; p=.04 ; R^{2}=.16$.

Neither sex $\left(\beta=.15 ; p=.35 ; R^{2}=.02\right)$, nor momentary mood changes ( $\operatorname{sad} \beta=.04 ; p=.84$; anxious, $\beta=-.02$; $p=.93 ; R^{2}=.02$ ), nor dispositional traits, (STAI, $\beta=.21$; $p=.50$; CES-D, $\beta=.05 ; p=.87$; STAXI-In, $\beta=.08$; $\left.p=.65 ; R^{2}=.06\right)$ were significantly associated with hemodynamic profile during mind wandering.

Results did not change when the analyses were performed replacing STAI and CES-D with PSQW and RRS, with these two variables not being significant predictors in any of the examined hierarchical regression models. Similar results were also obtained if anxious and sad were replaced by rumination and worry, with higher levels of rumination predicting a more vascular profile during the perseverative cognition $(\beta=.45 ; p=.01$; $\left.R^{2}=.11\right)$ and the problem solving induction $(\beta=.31 ; p=.03$; $R^{2}=.07$ ). Worry did not significantly add to the predictions (see the Limitation section for a possible explanation).

The absence of excessive multicollinearity was suggested by variance inflating factors not substantially greater than one and tolerance well above 0.2 .

\section{Discussion}

The present findings supported our hypotheses showing that perseverative cognition was associated with the same BP reactivity as more functional forms of repetitive thinking, but was uniquely characterized by a more vascular hemodynamic profile and (subsequently) delayed recovery.

Previous studies similarly showed that problem solving and worry elicited a cardiovascular reactivity of the same magnitude [5]. The present investigation extended such results with the inclusion of mind wandering as a comparison condition. Some authors previously reported an association between episodes of mind wandering and increases in heart rate, skin conductance [6, 7], and enhancement of the blink reflex [8]. However, when mind wandering was directly compared to or differentiated from perseverative cognition, its association with physiological reactivity disappeared both in laboratory [38] and in ambulatory studies [39-41]. These findings are underscored by the lower BP responses during the mind wandering induction in the current study.
Furthermore, we examined the hemodynamic correlates of functional and dysfunctional forms of repetitive thinking. Even if that was not the main objective of a previous study, the hemodynamic profile of angry rumination in comparison with a series of stressful tasks including a logical-mathematical task (which can be viewed as a form of problem solving) has been reported [10]. These findings are in line with our current results, in the way that the authors report a more vascular profile during rumination and a mixed profile during the logical-mathematical task. Here, we consistently found a vascular profile during perseverative cognition and a more myocardial profile during problem solving, as well as a mixed profile during mind wandering.

The present findings are in line with the Obrist's distinction of active versus passive coping [42]. In the Obrist view, active coping, which refers to an individual's attempts to exert personal control over environmental events, leads to significant beta-adrenergic influence on myocardial responses. However, such beta-adrenergic reactions become attenuated in situations that offer little opportunity to exercise instrumental control (i.e., passive coping), in which a significant vascular response is instead elicited. Problem solving can be considered as an example of active coping whereas perseverative cognition can be representative of passive coping. In our opinion, due to the nature of our tasks, it is possible to exclude that our results are due to quantitative differences in mental effort [5]. In terms of mental engagement, problem solving may be viewed as the most effortful condition but mind wandering (the default mode of operation of our brain) would be the least effortful or equal to perseverative cognition, making it difficult to interpret the present cardiovascular differences between these two experimental conditions solely in terms of mental effort. As a limit, this argument has not been demonstrated in this study in any empirical manner.

Drawing on Obrist's theory [42], Blascovich developed the biopsychosocial model of challenge and threat, according to which perceived challenge versus perceived threat reliably result in distinct patterns of physiological changes [43]. In this model, challenge is characterized by higher cardiac output and lower total peripheral resistance (a pattern similar to that taking place during aerobic exercise), whereas threat is characterized by the opposite pattern (i.e., higher total peripheral resistance and lower cardiac output). This view nicely fits with our finding of higher state anxiety being a significant predictor of a more vascular profile during perseverative cognition. In the Blascovich view, the threat cardiovascular pattern, which is characterized by arterial constriction rather than dilation, can result in strain on the coronary arteries, leading to damage, scarring, plaque deposits, and eventually ischemic heart disease or, if prolonged or repeated over time, hypertension. Indeed, persistent or excessive vasoconstriction is a pathognomonic indicator of hypertension $[9,12,13]$.

Keeping in mind the limitation that a 5 -min recovery period may not have been adequate in length [16], the second core 
result of the present study suggests that another crucial difference between functional and dysfunctional forms of repetitive thinking relies on the duration of the concomitant physiological activation. Perseverative cognition was in fact not only characterized by an increase in BP during the induction itself but also by a lack of BP recovery at the end of the task. Such sustained cardiovascular activation is not surprising as selfreported levels of rumination and worry during the recovery period suggest that participants were not able to stop perseverative cognition when asked to do so. This is of particular interest in light of evidence that hypertension is characterized by both elevated total peripheral resistance and delayed recovery $[9,44,45]$. It has further been suggested that recovery impairments may be among the earliest precursors to the development of essential hypertension in normotensive subjects at genetic risk of hypertension [46].

The effects of perseverative cognition on mood are one of the most well-replicated findings in this field [47] and do not need to be further commented.

When we looked at possible associations with dispositional traits, we found that a more vascular hemodynamic profile during perseverative cognition was predicted by higher levels of dispositional anger-in. Delayed recovery following anger provocation has been previously described and specifically linked to rumination [39-41]. More specifically, suppression of anger expression (i.e., anger-in) was specifically related to high BP, atherosclerosis, and delayed recovery [48-52]. A previous study found an association between anger-in levels and baroreceptor sensitivity during anger rumination [53], which is in line with the delayed recovery of BP that was seen in the present study after the perseverative cognition induction. Lastly, a vascular hemodynamic profile during angry rumination has been reported elsewhere [10], enhancing the robustness of the present results.

Some limitations need to be mentioned. First, the sample size was relatively small and may not have been adequate in some of the comparisons. Second, with a recovery period of adequate duration, we might have been able to capture the time needed for BP to fully recover after the perseverative cognition induction. Third, we experimentally induced perseverative cognition, mind wandering, and problem solving in the laboratory, whereas in the real world spontaneity is an intrinsic feature of these cognitive processes. This is particularly true for the mind wandering induction, whose investigation is commonly challenged by the lack of direct experimental control and its covert nature [54]. Among the methods generally used to investigate mind wandering, we preferred retrospective report to experience sampling to avoid the risk of altering the natural dynamics of the experience by periodically disrupting it [54]. A growing body of research employing resting state imaging measures and retrospective reports of mind wandering indicate that - in the absence of tasks requiring deliberative processing - the mind tends to wander [55,
56]. The retrospective report of mind wandering employed at the end of the induction has the advantage to preserve the integrity of time-course data and has been proven to be particularly suited to relate mind wandering to its physiological signatures, as suggested by studies using pupillometry [57] and EEG [58]. Directly related to this point, a further limitation of the present study is that we relied on post-task subjective reports (VAS) as the only measure of effectiveness for our inductions. Replication studies should include more objective measures of the distinct ongoing mental activities, such as EEG and test the possibility that rumination and worry have their own unique hemodynamic signatures. Our exploratory analysis seems to suggest that state rumination is a better predictor of hemodynamic profile than worry. This result should be, however, interpreted with caution especially considering that the Italian meaning of the terms used to measure state rumination and state worry are not exactly the same as in English. In Italian, the distinction between these two words does not exist in daily language; moreover, the word rumination encompasses threat (as confirmed with the significant correlation with state anxiety), whereas worry has a much milder meaning in terms of the evoked emotion compared to English. Lastly, despite the strength of the within-subject design used, and the counterbalancing of the order of the three inductions, and they may still have influenced each other in a non-natural way, each of them either enhancing or flattening the response to another. Our methodological approach could reduce the strength of the emotion experienced if some emotional episodes are not recalled as reliably and if the emotions are not relived as vividly.

Limitations notwithstanding, the present study is clinically relevant in that it provides further insights into the consequences of perseverative cognition for cardiovascular risk, furnishing information on its hemodynamics compared to more functional forms of repetitive thinking. Obviously, replication with a larger sample size, a wider range of inductions, and a longer recovery period is needed to test the robustness of the present findings.

\section{Compliance with Ethical Standards}

Funding Support for the last author was received from the Italian Ministry of Health Young Researcher Grant (2011-02348232).

Conflict of Interest The authors declare that they have no conflict of interest.

Ethical Approval All procedures performed in studies involving human participants were in accordance with the ethical standards of the institutional research committee and with the 1964 Helsinki declaration and its later amendments or comparable ethical standards.

Informed Consent Informed consent was obtained from all individual participants included in the study. 


\section{References}

1. Watkins ER. Constructive and unconstructive repetitive thought. Psychol Bull. 2008; 134: 163-206.

2. Brosschot JF, Gerin W, Thayer JF. The perseverative cognition hypothesis: a review of worry, prolonged stress-related physiological activation, and health. J Psychos Res. 2006; 60: 113-124.

3. Brosschot JF, Verkuil B, Thayer JF. Conscious and unconscious perseverative cognition: is a large part of prolonged physiological activity due to unconscious stress? J Psychosom Res. 2010; 69: 407-416.

4. Ottaviani C, Thayer JF, Verkuil B, et al. Physiological concomitants of perseverative cognition: a systematic review and meta-analysis. Psychol Bull. 2016; 142: 231-259.

5. Verkuil B, Brosschot JF, Borkovec TD, Thayer JF. Acute autonomic effects of experimental worry and cognitive problem solving: why worry about worry? Int J Clin Health Psychol. 2009; 9: 439453.

6. Smallwood J, Davies JB, Heim D, et al. Subjective experience and the attentional lapse: task engagement and disengagement during sustained attention. Conscious Cog. 2004; 13: 657-690.

7. Smallwood J, O'Connor RC, Sudberry MV, Haskell C, Ballantyne $\mathrm{C}$. The consequences of encoding information on the maintenance of internally generated images and thoughts: the role of meaning complexes. Conscious Cog. 2004; 13: 789-820.

8. Smilek D, Carriere JS, Cheyne JA. Out of mind, out of sight: eye blinking as indicator and embodiment of mind wandering. Psychol. Sci. 2010; 21: 786-789.

9. Julius S. Transition from high cardiac output to elevated vascular resistance in hypertension. Am Heart J. 1988; 116: 600-606.

10. Ottaviani C, Shapiro D, Goldstein IB, James JE, Weiss R. Hemodynamic profile, compensation deficit, and ambulatory blood pressure. Psychophysiology 2006; 43: 46-56.

11. Gregg ME, James JE, Matyas TA, Thorsteinsson EB. Hemodynamic profile of stress-induced anticipation and recovery. Int J Psychophysiol. 1999; 34: 147-162.

12. Mayet J, Hughes A. Cardiac and vascular pathophysiology in hypertension. Heart. 2003; 89: 1104-1109.

13. Sung BH, Lovallo WR, Pincomb GA, Wilson MF. Cardiac adaptation to elevated blood pressure in borderline hypertensive men. Am J Cardiol. 1993; 72: 407-412.

14. Panaite V, Salomon K, Jin A, Rottenberg J. Cardiovascular recovery from psychological and physiological challenge and risk for adverse cardiovascular outcomes and all-cause mortality. Psychosom Med. 2015; 77: 215-226.

15. Ottaviani C, Shapiro D, Goldstein IB, Mills PJ. Vascularprofile, delayed recovery, inflammatory process, and ambulatory blood pressure: laboratory-to-life generalizability. Int J Psychophysiol. 2007; 66: 5665 .

16. Steptoe A, Marmot M. Impaired cardiovascular recovery following stress predicts 3-year increases in blood pressure. J Hypertens. 2005; 23: 529-536.

17. Ring C, Burns VE, Carroll D. Shifting hemodynamics of blood pressure control during prolonged mental stress. Psychophysiology 2002; 39: 585-590.

18. Rutledge T, Hogan BE. A quantitative review of prospective evidence linking psychological factors with hypertension development. Psychosom Med. 2002; 64: 758-766.

19. Jennings JR, Kamarck T, Stewart C, Eddy M, Johnson P. Alternate cardiovascular baseline assessment techniques: vanilla or resting baseline. Psychophysiology. 1992; 29: 742-750

20. Eckert S, Horstkotte D. Comparison of Portapres non-invasive blood pressure measurement in the finger with intra-aortic pressure measurement during incremental bicycle exercise. Blood Press Monit. 2002; 7: 179-183.

21. Gregg ME, Matyas TA, James JE. A new model of individual differences in hemodynamic profile and blood pressure reactivity. Psychophysiology 2002; 39: 64-72.

22. Guyton A. Human physiology and mechanisms of disease (4th ed). Philadelphia: W.B. Saunders; 1987.

23. Nolen-Hoeksema S, Morrow J. A prospective study of depression and posttraumatic stress symptoms after a natural disaster: the 1989 Loma Prieta Earthquake. J Pers Soc Psychol. 1991; 61:115-121.

24. Nolen-Hoeksema S, Parker LE, Larson J. Ruminative coping with depressed mood following loss. J Pers Soc Psychol. 1994; 67:92-104.

25. Nolen-Hoeksema S, Davis CG. "Thanks for sharing that": ruminators and their social support networks. J Pers Soc Psychol. 1999; 77: 801-14.

26. Meyer TJ, Miller ML, Metzger RL, Borkovec TD. Development and validation of the Penn State Worry Questionnaire. Behav. Res. Ther. 1990; 28: 487-495.

27. Stober J. Reliability and validity of two widely-used worry questionnaires: self-report and self-peer convergence. Pers Individ Dif. 1998; 24: 887-890.

28. Molina S, Borkovec TD. The Penn State Worry Questionnaire: psychometric properties and associated characteristics. In: Davey GCL, Tallis F, eds. Worrying: perspectives on theory, assessment and treatment. New York, NY: Wiley; 1994: 265-283.

29. Brown TA, Marten PA, Barlow DH. Discriminant validity of the symptoms comprising the DSM-III-R and DSM-IV associated symptom criterion of generalized anxiety disorder. J Anxiety Disord. 1995; 9: 317-328.

30. Spielberger CD, Gorsuch RL, Lushene RE. STAI Manual. Palo Alto, CA: Consulting Psychologists Press; 1970.

31. Spielberger CD. State-trait anxiety inventory: bibliography (2nd ed.). Palo Alto, CA: Consulting Psychologists Press, 1989.

32. Spielberger CD, Gorsuch RL, Lushene R, Vagg PR, Jacobs GA. Manual for the state-trait anxiety inventory. Palo Alto, CA: Consulting Psychologists Press, 1983.

33. Radloff LS. The CES-D scale: a self-report depression scale for research in the general population. Appl. Psychol. Meas. 1977; 1: 385-401.

34. Carleton RN, Thibodeau MA, Teale MJN, Welch PG, Abrams MP, Robinson T, Asmundson GJ. (2013) The Center for Epidemiologic Studies Depression Scale: A Review with a Theoretical and Empirical Examination of Item Content and Factor Structure. PLoS One 2013; 8: e58067.

35. Spielberger CD, Johnson EH, Russell SF, Crane RJ, Jacobs GA, Worden TJ. The experience and expression of anger: construction and validation of an anger expression scale. In: Chesney MA, Rosenman RH, eds. Anger and hostility in cardiovascular and behavioral disorders. Washington DC: Hemisphere; 1985: 5-30.

36. Spielberger CD, Reheiser EC. Measuring anxiety, anger, depression, and curiosity as emotional states and personality traits with the STAI, STAXI, and STPI. In: Hersen M, Segal DL, Hilsenroth M, eds. Comprehensive Handbook of Psychological Assessment (Vol. 2): Personality Assessment. New York: Wiley; 2004: 74-80.

37. James JE, Gregg ME. Hemodynamic effects of dietary caffeine, sleep restriction, and laboratory stress. Psychophysiology 2004; 41: 914-923.

38. Ottaviani C, Shapiro D, Couyoumdjian A. Flexibility as the key for somatic health: from mind wandering to perseverative cognition. Biol Psychol. 2013; 94: 38-43.

39. Ottaviani $\mathrm{C}$, Couyoumdjian A. Pros and cons of a wandering mind: a prospective study. Front Psychol. 2013; 4: 524, DOI: 10.3389 /fpsyg.2013.00524.

40. Ottaviani C, Medea B, Lonigro A, Tarvainen M, Couyoumdjian A. Cognitive rigidity is mirrored by autonomic inflexibility in daily life perseverative cognition. Biol Psychol. 2015; 107: 24-30. 
41. Ottaviani C, Shahabi L, Tarvainen M, Cook I, Abrams M, Shapiro D. Cognitive, behavioral, and autonomic correlates of mind wandering and perseverative cognition in major depression. Front Neurosci. 2015; 8: 433. doi: 10.3389/fnins.2014.00433

42. Obrist PA. Cardiovascular psychophysiology. New York: Plenum; 1981.

43. Blascovich J. Challenge, threat, and health. In: Shah JY, Gardner WL, eds. Handbook of motivation science. New York: Guilford; 2008: 481-493.

44. Schuler JL, O'Brien WH. Cardiovascular recovery from stress and hypertension risk factors: a meta-analytic review. Psychophysiology. 1997; 34: 649-659.

45. Steptoe A, Donald AE, O'Donnell K, Marmot M, Deanfield JE. Delayed blood pressure recovery after psychological stress is associated with carotid intima-media thickness: Whitehall psychobiology study. Arterioscler Thromb Vasc Biol. 2006; 26: 2547-2551.

46. Schneider GM, Jacobs DW, Gevirtz RN, O'Connor DT. Cardiovascular haemodynamic response to repeated mental stress in normotensive subjects at genetic risk of hypertension: evidence of enhanced reactivity, blunted adaptation, and delayed recovery. $\mathrm{J}$ Hum Hypertens. 2003; 17: 829-840.

47. Nolen-Hoeksema S, Wisco BE, Lyubomirsky S. Rethinking rumination. Perspect Psychol Sci. 2008; 3: 400-424.

48. Gerin W, Davidson KW, Christenfeld NJ, Goyal T, Schwartz JE. The role of angry rumination and distraction in blood pressure recovery from emotional arousal. Psychosom Med. 2006; 68: 64-72.

49. Ottaviani C, Shapiro D, Fitzgerald L. Rumination in the laboratory: what happens when you go back to everyday life? Psychophysiology. 2011; 48: 453-461
50. Suchday S, Carter MM, Ewart CK, Larkin KT, Desiderato O. Anger cognitions and cardiovascular recovery following provocation. J Behav Med. 2004; 27: 319-341.

51. al'Absi M, Bongard S. Neuroendocrine and behavioral mechanisms mediating the relationship between anger expression and cardiovascular risk: assessment considerations and improvements. J Behav Med. 2006; 29: 573-591.

52. Vella EJ, Friedman BH. Hostility and anger in: cardiovascular reactivity and recovery to mental arithmetic stress. Int $\mathrm{J}$ Psychophysiol. 2009; 72: 253-259.

53. Ottaviani C, Shapiro D, Davydov DM, Goldstein IB, Mills PJ. The autonomic phenotype of rumination. Int J Psychophysiol. 2009; 72: 267-275.

54. Smallwood J, Schooler JW. The science of mind wandering: empirically navigating the stream of consciousness. Annu Rey Psychol. 2015; 66: 487-518.

55. Fox KC, Spreng RN, Ellamil M, Andrews-Hanna JR, Christoff K. The wandering brain: meta-analysis of functional neuroimaging studies of mind-wandering and related spontaneous thought processes. Neuroimage. 2015; 111: 611-621.

56. Mason MF, Norton MI, Van Horn JD, Wegner DM, Grafton ST, Macrae CN. Wandering minds: the default network and stimulusindependent thought. Science 2007; 315: 393-395.

57. Smallwood J, Brown KS, Baird B, Mrazek MD, Franklin MS, Schooler JW. Insulation for daydreams: a role for tonic norepinephrine in the facilitation of internally guided thought. PLoS One 2012; 7:e33706.

58. Barron E, Riby LM, Greer J, Smallwood J. Absorbed in thought: the effect of mind wandering on the processing of relevant and irrelevant events. Psychol Sci. 2011; 22: 596-601. 